\title{
NOTES
}

\section{Preparation of Polypyrrole-Poly(vinyl chloride) Composite Films by Interphase Oxidative Polymerization}

\author{
Masakazu NaKata and Hideo KISE \\ Institute of Materials Science, University of Tsukuba, Tsukuba, Ibaraki 305, Japan
}

(Received May 20, 1992)

\begin{abstract}
KEY WORDS Polypyrrole / Poly(vinyl chloride) / Composite Film / Interphase Polymerization / Oxidative Polymerization / Electrical Conductivity /
\end{abstract}

Polypyrrole (PPy) is a conducting polymer which has attracted much attention because of its high stability under ambient conditions together with high electrical conductivity. This may allow various applications, such as electrochromic displays, ${ }^{1,2}$ modified electrode catalysts, ${ }^{3}$ and shielding material for electromagnetic field. ${ }^{4}$ PPy films are normally prepared by electrochemical polymerization of pyrrole on electrodes. ${ }^{5}$ The oxidative (chemical) polymerization of pyrrole has also been known for many years, ${ }^{6,7}$ which in general produces PPy powders. Recently, a method of interphase oxidative polymerization of pyrrole has been developed which produces PPy films at the interphase between an aqueous solution of an oxidant and organic solution of pyrrole. ${ }^{8-11}$

The main drawback of PPy is poor mechanical properties as indicated by its low elongation at break and its brittleness, ${ }^{12,13}$ and this restricts its widespread technological applications. In order to overcome this problem, it has been demonstrated that conductive composites of PPy with polymer materials can be prepared by chemical or electrochemical methods. For example, composites with poly(vinyl alcohol) (PVA) ${ }^{14}$ methylcellulose,${ }^{15}$ polyacrylonitrile, ${ }^{16}$ poly $(p$ phenyleneterephthalamide), ${ }^{17}$ polyimide, ${ }^{18}$ and poly(ethylene terephthalate) ${ }^{19,20}$ have been prepared.

Composite films of PPy with poly(vinyl chloride) (PVC) have also been prepared by electrochemical polymerization of pyrrole on electrodes covered with PVC cast films. ${ }^{13,21-23}$ It was reported that the composite films thus obtained had mechanical properties very similar to those of pure PVC. ${ }^{13}$ In this article, we report a new and facile method of preparation of composite films of PPy with PVC by interphase oxidative polymerization of pyrrole between an aqueous oxidant solution and an organic pyrrole solution separated by a PVC film.

\section{EXPERIMENTAL}

Pyrrole monomer was purchased from Tokyo Kasei Kogyo Co. and purified by distillation under reduced pressure. Anhydrous iron(III) chloride from Wako Pure Chemical Industries, Ltd. was used without further purification. Porous PVC films were generously supplied from Mitsubishi Petrochemical Co., Ltd. ${ }^{24}$ The thickness and density of the films were $90-100 \mu \mathrm{m}$ and $0.60-0.64 \mathrm{gcm}^{-3}$, respectively.

A typical procedure of preparation of PVC-PPy composite film is as follows: a PVC 


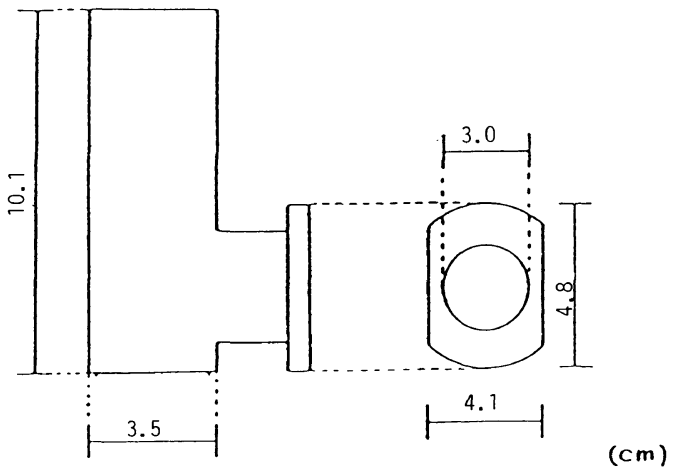

Figure 1. Glass tube apparatus for interphase polymerization.

film was placed vertically between a couple of glass tubes shown in Figure 1, and they were fixed by double clips. A solution of pyrrole in benzene and an aqueous solution of iron(III) chloride were placed in each of the tubes; the two solutions were separated by the PVC film. The reactants diffuse across the film and pyrrole was polymerized in or at the surface of the film. After the reaction, PVC-PPy composite film was washed with water and methanol, and dried under vacuum for more than $24 \mathrm{~h}$ at $45^{\circ} \mathrm{C}$. Electrical conductivity of the film was measured using four-probe method under vacuum at $30^{\circ} \mathrm{C}$.

\section{RESULTS AND DISCUSSION}

The interphase oxidative polymerization of pyrrole has been extensively studied using aqueous solutions of iron(III) salts and organic solutions of pyrrole. ${ }^{10,11}$ It was found that the nature of anions in iron salts strongly influences the conductivity of the polypyrrole (PPy) films. Among the iron salts used, iron chloride and perchlorate are the best with respect to the electrical conductivity of the resultant PPy films. It has been reported that when iron(III) chloride is used as an oxidant, chloride ion is incorporated in PPy as a dopant and plays an important role in the conducting process. ${ }^{9,25}$

The selection of organic solvents is also crucial for obtaining PPy films with good
Table I. Preparation of PPy-PVC composite films. ${ }^{\mathrm{a}}$

\begin{tabular}{|c|c|c|c|}
\hline \multirow{2}{*}{$\frac{\text { Pyrrole conc. }}{M}$} & \multicolumn{2}{|c|}{ Thickness $/ \mu \mathrm{m}$} & \multirow{2}{*}{$\frac{\text { Conductivity }}{\mathrm{S} \mathrm{cm}^{-1}}$} \\
\hline & A & B & \\
\hline 0.69 & 92 & 146 & 1.88 \\
\hline 0.40 & 95 & 155 & 0.92 \\
\hline 0.13 & 95 & 89 & 0.25 \\
\hline
\end{tabular}

${ }^{a} \mathrm{FeCl}_{3}(2.6 \mathrm{M})$ in water, pyrrole in benzene, room temperature for $4 \mathrm{~h}$. A, before reaction; B, after reaction.

mechanical properties and high conductivity; benzene or toluene were suitable for this purpose. Therefore, we studied the preparation of PPy-PVC composite films using an aqueous solution of iron(III) chloride and a pyrrole solution in benzene. Typical results are shown in Table I. Under these conditions, growth of colored PPy was observed only on aqueous side of PVC films without significant coloration on organic side of PVC. No precipitate was formed in both solutions. However, when the concentration of iron chloride was lowered, for example to $0.7 \mathrm{M}$, small amounts of colored precipitates were formed in the aqueous solutions. This suggests that part of pyrrole was dissolved in aqueous solution before being polymerized due to lower concentration of the oxidant.

These results indicate that, under these conditions, pyrrole diffused through PVC film from organic phase to aqueous phase and was polymerized on or near the surface of the PVC film to afford two-layered composite films. PVC is one of the hydrophobic polymers, and diffusion of iron ion across the PVC film may be inhibited because of hydrophilic nature of iron ions.

On the basis of the above consideration, we tried to change the hydrophobic/hydrophilic nature of the solution of iron chloride in order to obtain homogeneous (single-layered) PPyPVC composite films. Methanol was chosen as a component of mixed solvent of iron chloride to increase the diffusivity of iron ion into PVC 


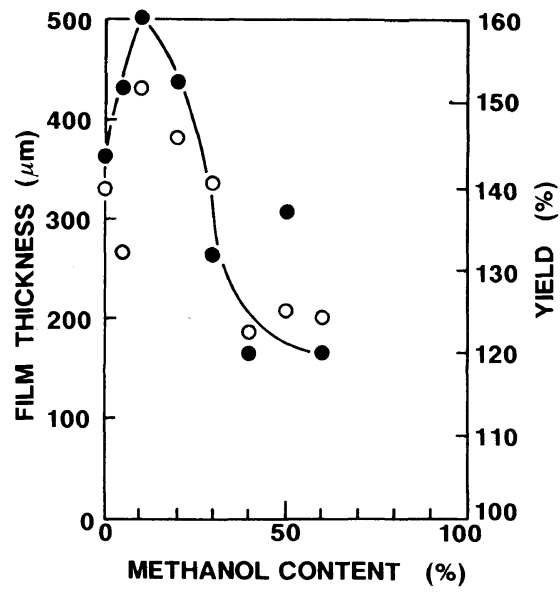

Figure 2. Effects of methanol content on thickness $(O)$ and yield (O) of PPy-PVC films: Yield $(\%)=$ (weight of $\mathrm{PPy}-\mathrm{PVC}) /($ weight of $\mathrm{PVC}) \times 100 . \mathrm{FeCl}_{3} 2.4 \mathrm{M}$, pyrrole $0.69 \mathrm{M}$, room temperature, reaction time $4 \mathrm{~h}$.

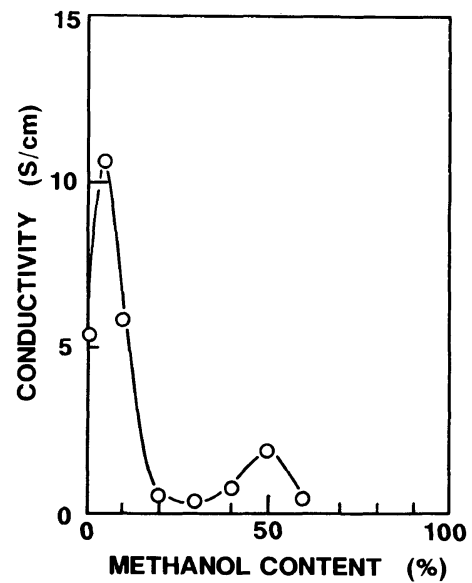

Figure 3. Effects of methanol content on conductivity: $\mathrm{FeCl}_{3} 2.4 \mathrm{M}$, pyrrole $0.69 \mathrm{M}$, room temperature, reaction time $4 \mathrm{~h}$.

films. It was found that by the addition of methanol to the aqueous iron chloride solution, coloration occurred on both sides of PVC films, which indicates the formation of PPy in the PVC films. At or above $40 \%$ methanol, coloration of the organic solution was observed which indicates that a part of pyrrole was polymerized in the organic solution. This may be indication of the diffusion of iron ion through the PVC film owing to increase in hydrophobicity of the
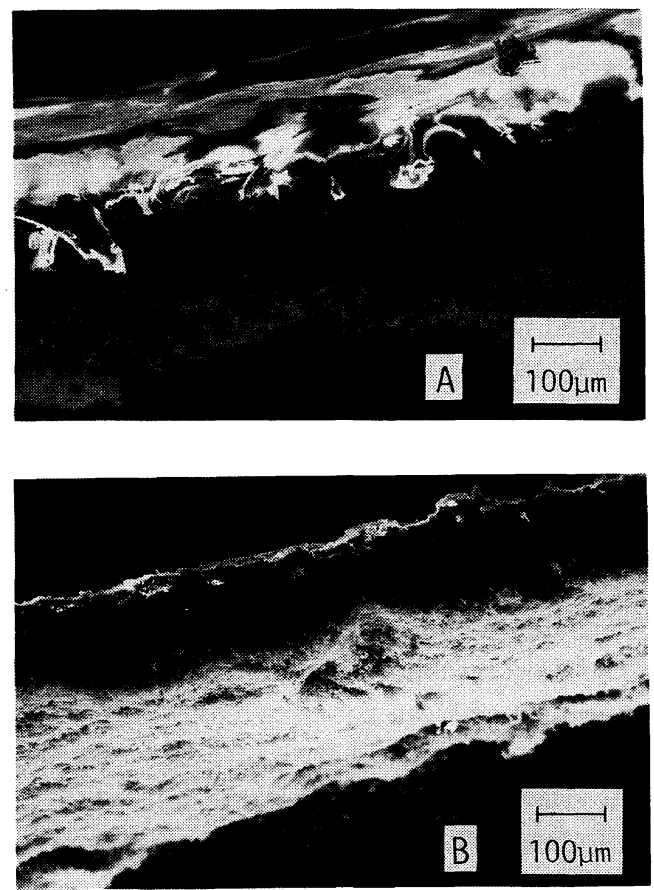

Figure 4. SEM of cross section of PPy-PVC films. A, methanol $20 \%$; B, methanol $60 \%$.

oxidant solvent.

Figure 2 shows the effects of methanol content on thickness and yield of the composite films. Both the thickness and yield of the films exhibit maxima at about $10 \%$ methanol, then decrease sharply with increase in methanol content. This may be a consequence of the formation of dense PPy in the pores of PVC films which would retard the diffusion of both pyrrole and iron ion. The effect of methanol content on electrical conductivity is shown in Figure 3. Similar to the results in Figure 2, maximum conductivity was obtained at about $5 \%$ methanol; at higher methanol content, conductivity dropped sharply. It may be assumed that at higher methanol content, the formation of PPy predominates in PVC films where PPy is segregated as compared to continuous layer of PPy on the surface of PVC. This would cause decrease in the average conductivity of the films.

The analysis of the cross section of the films 
obtained using $20 \%$ or lower methanol contents indicates the formation of two-layered composite films of PPy and PVC (Figure 4A). PPy was grown on the aqueous side of the PVC film (the upper layer in Figure 4A is PVC and the lower layer is PPy). At $60 \%$ methanol content, films of single-layered structure were obtained (Figure 4B).

In summary, PPy-PVC conductive composite films were prepared by the interphase polymerization of pyrrole between aqueous solutions of iron(III) salts and organic solutions of pyrrole separated by PVC films. The structure of the composite films can be changed by changing the nature of the solvents of the oxidant; films of two-layered structure were obtained from aqueous iron solutions and films of single-layered structure were obtained from water-methanol mixed solvents. The method described here for PVC can be applied to the preparation of composite films of PPy with other porous or swelling materials by easy operation in simple reaction apparatus.

\section{REFERENCES}

1. R. Bjorklund, S. Andersson, S. Allenmark, and I. Lundstrom, Mol. Cryst. Liq. Cryst., 121, 263 (1985).

2. S. Kuwabata, H. Yoneyama, and H. Tamura, Bull. Chem. Soc. Jpn., 57, 2247 (1984).

3. A. Deronzier and J.-C. Moutet, Acc. Chem. Res., 22, 249 (1989).

4. K. Yoshino, M. Tabata, and T. Osawa, J. Jpn. Chem. Soc., 342 (1986).

5. K. K. Kanazawa, A. F. Diaz, W. D. Gill, P. M.
Grant, G. B. Street, G. P. Gardini, and J. F. Kwak, Synth. Met., 1, 329 (1979/80).

6. K. C. Khulbe, R. S. Mann, and C. P. Khulbe, J. Polym. Sci. Polym. Chem. Ed., 20, 1089 (1982).

7. N. Mermilliod, J. Tanguy, and F. Petiot, J. Electrochem. Soc., 133, 1073 (1986).

8. V. Bocchi and G. P. Gardini, J. Chem. Soc., Chem. Commun., 148 (1986).

9. T. H. Chao and J. March, J. Polym. Sci., A: Polym. Chem., 26, 743 (1988).

10. M. Nakata, Y. Shiraishi, M. Taga, and H. Kise, Makromol. Chem., 193, 765 (1992).

11. M. Nakata, M. Taga, and H. Kise, Polym. J., 24, 437 (1992).

12. A. F. Diaz and B. Hall, IBM J. Res. Dev., 27, 342 (1983).

13. M.-A. De Paoli, R. J. Waltman, A. F. Diaz, and J. Bargon, J. Polym. Sci. Polym. Chem. Ed., 23, 1687 (1985).

14. T. Ojio and S. Miyata, J. Jpn. Chem. Soc., 348 (1986).

15. R. B. Bjorklund and B. Liedberg, J. Chem. Soc., Chem. Commun., 1293 (1986).

16. N. V. Bhat and E. Sundaresan, J. Appl. Polym. Sci., 38, 1173 (1989).

17. K. Koga, T. Iino, S. Ueta, and M. Takayanagi, Polym. J., 21, 303 (1989).

18. B. Tieke and W. Gabriel, Polymer, 31, 20 (1990).

19. T. Yoshikawa, S. Machida, T. Ikegami, A. Techagumpuch, and S. Miyata, Polym. J., 22, 1 (1990).

20. C. Li and Z. Song, Synth. Met., 40, 23 (1991).

21. O. Niwa and T. Tamamura, J. Chem. Soc., Chem. Commun., 817 (1984).

22. M.-A. De Paoli, R. J. Waltman, A. F. Diaz, and J. Bargon, J. Chem. Soc., Chem. Commun., 1015(1984).

23. K. Uosaki, K. Okazaki, and H. Kita, J. Polym. Sci., A: Polym. Chem., 28, 399 (1990).

24. S. Hirose, A. Shimizu, and T. Nose, J. Appl. Polym. Sci., 23, 3193 (1979).

25. S. Machida, S. Miyata, and A. Techagumpuch, Synth. Met., 31, 311 (1989). 